\title{
NOMA-enabled Optimization Framework for Next-generation Small-cell IoV Networks under Imperfect SIC Decoding
}

\author{
Wali Ullah Khan, Xingwang Li, Asim Ihsan, Mohammad Ayoub Khan, Varun G Menon, and Manzoor Ahmed
}

\begin{abstract}
To meet the demands of massive connections, diverse quality of services (QoS), ultra-reliable and low latency in the future sixth-generation (6G) Internet-of-vehicle (IoV) communications, we propose non-orthogonal multiple access (NOMA)enabled small-cell IoV network (SVNet). We aim to investigate the trade-off between system capacity and energy efficiency through a joint power optimization framework. In particular, we formulate a nonlinear multi-objective optimization problem under imperfect successive interference cancellation (SIC) detecting. Thus, the objective is to simultaneously maximize the sum-capacity and minimize the total transmit power of NOMA-enabled SVNet subject to individual IoV QoS, maximum transmit power and efficient signal detecting. To solve the nonlinear problem, we first exploit a weighted-sum method to handle the multi-objective optimization and then adopt a new iterative Sequential Quadratic Programming (SQP)-based approach to obtain the optimal solution. The proposed optimization framework is compared with Karush-Kuhn-Tucker (KKT)-based NOMA framework, average power NOMA framework, and conventional OMA framework. Monte Carlo simulation results unveil the validness of our derivations. The presented results also show the superiority of the proposed optimization framework over other benchmark frameworks in terms of system sum-capacity and total energy efficiency.
\end{abstract}

Index Terms-6G, energy efficiency, multi-objective optimization, non-orthogonal multiple access, sum-capacity, sequential quadratic programing.

\section{INTRODUCTION}

$\mathbf{R}$ ECENT years have witnessed a great increase in the applications of vehicular networks to improve traffic efficiency, reliability, control, and passenger safety [1], [2]. Internet-of-vehicle (IoV) has emerged as a key enabler technology of the future intelligent transportation systems to increase the existing capabilities of vehicular ad-hoc networks with Internet-of-things [3], [4]. Communication of IoV involves vehicle-to-roadside (V2R), vehicle-to-vehicle (V2V), vehicle-to-pedestrians (V2P), vehicle-to-infrastructure (V2I),

W. U. Khan is with the Interdisciplinary Centre for Security, Reliability and Trust, SigCom, University of Luxembourg, Luxembourg. (email: waliullahkhan30@gmail.com)

$\mathrm{X}$. Li is with the School of Physics and Electronic Information Engineering, Henan Polytechnic University, Jiaozuo, China (email: lixingwangbupt@gmail.com)

A. Ihsan is with with Department of Information and Communication Engineering, Shanghai Jiao Tong University, Shanghai 200240, China. (email: ihsanasim@sjtu.edu.cn)

M. A. Khan is with the College of Computing and Information Technology, University of Bisha, Bisha, 67714, Saudi Arabia. (email: ayoub.khan@ieee.org)

V. G Menon is with the SCMS School of Engineering and Technology, 488071 Ernakulam, Kerala 683 576, India. (e-mail: varunmenon@ieee.org)

M. Ahmed is with the College of Computer Science and Technology, Qingdao University, Qingdao 266071, China. (e-mail: manzoor.achakzai@gmail.com) vehicle-to-bicycle (V2B), and vehicle-to-sensors (V2S) [5], [6]. The sixth-generation (6G) and beyond IoV networks are expected to support large-scale wireless connections with high data rates and lower energy consumption [7]. Non-orthogonal multiple access (NOMA) has been raised as a promising air interface technology that allows multiple IoVs to communicate over the same time/frequency resources, and thus can significantly increase the number of served IoVs [8]. It is different from the traditional orthogonal multiple access (OMA) where one frequency resource can only accommodate one $\mathrm{IoV}$ at one time. By using the superposition coding technique, NOMA first superimposes the data of multiple IoVs over the same frequency with different transmission power [9]. Then, IoVs adopt successive interference cancellation (SIC) to decode their received signals [10]. More specifically, IoV with the larger value of channel gain is assigned less transmit power compared to those IoVs with smaller values of channel gains [11]. Thus, IoV with the larger value of channel gain can apply SIC and subtract the interference of other IoVs with smaller values of channel gains [12]. In the end, an IoV with the lowest value of channel gain cannot subtract the interference and treat it as noise [13]. Moreover, NOMA improves not only the sum-capacity but also guarantees system fairness [14].

\section{A. Related Work}

NOMA-enabled vehicular communication has been studied by both industry and academia in recent years. To maximize the minimum rate of vehicles in vehicle-to-everything (V2X) systems, Zheng et al. [15] provided a joint optimization framework of user clustering, channel assignment, and power loading to improve the fairness of the NOMA system, which was also subjected to a minimum QoS requirement. The efficient solutions were obtained using matching theory, perron frobenius theorem, and kuhn munkres algorithm. Wang et al. [16] presented a centralized resource management framework to maximize the total capacity of device-to-device (D2D)aided V2X networks. The authors used a three-partite interference hyper-graph-based greedy, iterative, and matching algorithm for resource allocation to manage the complex interference in the system. Reference [17] proposed a decentralized V2X network supporting full-duplex NOMA for both crowded and urban scenarios. They derived approximate closed-form solutions and their objective was to enhance the system capacity with different QoS requirements. To reduce the complexity and improve the achievable capacity of V2X communication, Xiao et al. [18] provided the efficient vehicle clustering and power control framework for NOMA-enabled V2X networks under vehicle QoS constraint. The authors of 
[19] and [20] maximized the sum-capacity of D2D-aided V2X communication using the centralized resource management frameworks. They used the interference hyper-graph method to efficiently manage the complex interference of the system. The problem of joint optimization was investigated by Qian et al. [21] to maximize the sum-rate of vehicle-to-small networks. The problem of efficient cell association and power control was first transformed and then solved using standard gradient scheduling and hierarchical power control algorithms. The work of [22] proposed a resource allocation framework to optimize the spectral efficiency and received packet ratio of multiple-input multiple-output V2V networks.

Moreover, the authors of [23] and [24] explored the resource management problems of V2X networks. The objectives were to reduce latency and improve the reliability of the systems. They first performed source-destination selection and spectrum allocation using a centralized semi-persistent algorith$\mathrm{m}$. Then, they employed an iterative distributed algorithm for efficient power allocation. The works of [25] and [26] proposed resource allocation problems for NOMA-enabled vehicular heterogeneous networks. Their objective was to improve the spectral efficiency and reliability of the system through efficient spectrum allocation and power control. In [27], Jaiswal et al. derived closed-form expressions of NOMAenabled vehicular communication networks to investigate outage probability, bit-error-rate and ergodic capacity. Reference [28] considered full-duplex V2X communication to improve the ergodic capacity through an efficient selection of roadside units (RSUs). Abbasi et al. [29] presented a cooperative V2X communication, where unmanned-aerial-vehicle acted as an amplify-and-forward relay. The objective was to maximize the sum-rate through efficient trajectory planning and power control. The authors of [30] derived exact expressions for outage probability in cognitive-based V2X communications. They adopted an efficient RSU selection scheme and considered the constraint of transmit power. Khan et al. [31] investigated the trade-off between sum-capacity and energy efficiency of V2I networks. The problem of power allocation was solved through Karush-Kuhn-Tucker (KKT) conditions and the QoS of vehicles were satisfied. Besides, the work of [32] also maximized the energy efficiency of V2X communication. They adopted the Dinkelback method and dual theory to handle the nonconvex energy efficiency problem. Of late, the work of [33] also investigated a joint optimization framework backscatterenabled NOMA V2X communication. Their objective was to maximize the sum rate of the network subject to minimum rate requirements. The optimization problem was first transformed from non-convex to a convex one, and then solved through KKT conditions, where Lagrangian multipliers are iteratively computed using sub-gradient method.

\section{B. Motivation and Contributions}

Most of the above literature has considered the perfect SIC decoding process at IoVs. However, an error can occur during the decoding process of signal in practical NOMA systems. In such a situation, the IoV with the larger value of channel gain cannot subtract the interference of other IoVs with smaller values of channel gains, causing significant performance degradation in system performance. Besides, the focus of many studies is on single-objective optimization frameworks either maximize the sum-capacity or energy efficiency. To the best of the author's knowledge, an optimization framework that jointly maximizes the sum-capacity and total energy efficiency of IoVs under imperfect SIC decoding has not yet been investigated. To bridge this gap, we aim to propose a joint optimization framework to investigate a trade-off on sumcapacity and total energy efficiency of NOMA-enabled smallcell IoV network (SVNet) under imperfect SIC decoding. An optimal solution is presented using a sequential quadratic programming (SQP) approach, where the quality of services (QoS) of IoVs are satisfied. Obtained results confirm the superiority of the proposed optimization framework over the benchmark optimization frameworks. The important contributions of our paper are summarized as follows.

- We propose a joint optimization framework for nextgeneration NOMA-enabled SVNet to simultaneously maximize the sum-capacity and total energy efficiency. To improve the spectral efficiency, we assume that all small-cell access points (SAPs) share the same spectrum resources at a given time. Designing a more practical system, we formulate a nonlinear multi-objective optimization problem under the consideration of imperfect SIC decoding. In particular, we jointly maximize the sumcapacity and minimize the total transmit power of NOMA SVNet while taking the constraints of minimum QoS, maximum transmit power, and efficient signal decoding into account.

- To obtain an optimal solution to the non-convex power allocation problem, we first exploit the weightedsum method to handle the multi-objective problem and then adopt a new optimization approach based on sequential quadratic programming (SQP) for optimal power allocation. SQP is an iterative approach, where the nonlinear/non-convex optimization problem is modeled by adopting quadratic optimization subproblems. We also provide the comparison of the proposed optimization framework with the KKT approach, average power allocation, and conventional OMA approach.

- The Monte Carlo simulation results provide an extensive comparison of the proposed optimization framework with benchmark NOMA SVNet, average power NOMA SVNet, and conventional OMA SVNet, respectively. Presented results reveal the superiority of the proposed NOMA-enabled SVNet approach over the other optimization frameworks in terms of sum-capacity and total energy efficiency. It is also found that the performance of the proposed framework degrades as the value imperfect SIC parameter increases.

The remainder of this paper can be organized as follow: Section II provides the system model of NOMA-enabled SVNet and discusses its different steps involved in the problem formulation. Section III derives the optimal solution of NOMAenabled SVNet based on the SQP approach and suboptimal solution based on the KKT approach. Section IV presents the simulation results and discussion of the proposed optimization 


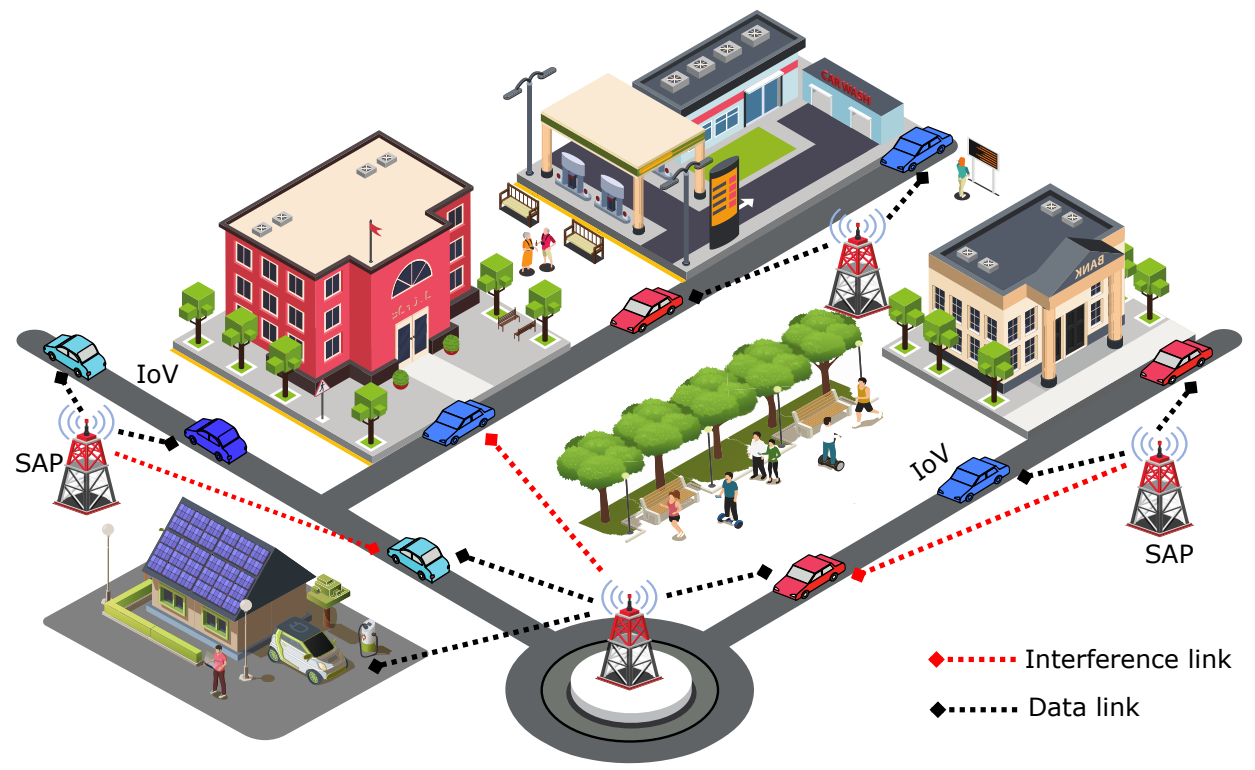

Fig. 1: System model of NOMA SVNet.

framework in comparison with other benchmark frameworks. Finally, Section V concludes this work with some future research directions.

\section{System Model and Problem Formulation}

A downlink transmission of SVNet is considered as depicted in Fig. 1. In each small-cell, and SAP serves multiple IoVs using NOMA protocol while different SAPs are considered to utilize OMA protocol. We denote the set of small-cells as $\mathcal{M}=\{m \mid 1,2, \ldots, M\}$, where $m$ is the index of the small-cell access point (SAP) $m$. Each SAP is located at the roadside and serves a set of Internet-of-vehicles (IoVs) using NOMA protocol. The set of IoVs serve by SAP $m$ can be denoted as $\mathcal{N}=\{n \mid 1,2, \ldots, N\}$, where $n$ shows the index of the IoV $n^{1}$. We assume that: i) All the SAPs utilize the same spectrum resources such that they produce co-channel interference to each other; ii) All the devices in the network are equipped with omnidirectional antenna; iii) The channel state information (CSI) of all IOVs are available at SAPs [34]; vi) The channels between SAPs and IoVs are independent and undergo Rayleigh fading [35].

During the communication process, each SAP needs to transmit different pieces of information to its serving IoVs over the same spectrum resource at the same time. To do so, SAPs apply the NOMA protocol to transmit different signals to their associated IoVs. Thus, IoVs associated with the same SAP not only have interference due to NOMA, known as intracell interference but also receive interference from neighboring SAPs due to co-channel, also called inter-cell interference. According to the NOMA principle, interference from IoV with weak channel conditions can be successfully removed at IoV with strong channel conditions using the SIC decoding

\footnotetext{
${ }^{1}$ In this work, we consider that the vehicle association with SAPs is accomplished before the joint optimization framework. Efficient vehicle association can further enhance the performance of SVNet, however, is set aside for future research work.
}

technique. The removal of this interference can be successful if the channel to inter-cell interference plus noise ratio (CINR) of the IoV with stronger channel conditions is greater than the CINR of the IoV with weak channel conditions. With no loss of generality, we assume that the CINR of different IoVs associated with SAP $m$ are sorted as

$$
\frac{\left|g_{n, m}\right|^{2}}{\sigma^{2}+\left|g_{n, m}\right|^{2} \Theta_{n, m}} \leq \frac{\left|g_{n-1, m}\right|^{2}}{\sigma^{2}+\left|g_{n-1, m}\right|^{2} \Theta_{n-1, m}}
$$

where $\left|g_{n, m}\right|=\sqrt{h_{n, m}, r_{n, m}^{-\epsilon}}$ denotes the channel coefficient between IoV $n$ and SAP $m$ such that $r_{n, m}$ is the distance between IoV $n$ and SAP $m, \epsilon$ represents the path-loss exponent, and $h_{n, m}$ is the channel gain between IoV $n$ and SAP $m$, respectively. The term $\Theta_{n, m}=\sum_{m^{\prime}=1}^{M} \sum_{n^{\prime}=1}^{N} p_{n^{\prime}, m^{\prime}}$ is the interference power from neighboring SAPs and $\sigma^{2}$ is the variance of additive white Gaussian noise (AWGN). According to the NOMA protocol, the power allocation at SAP $m$ for IoV $n$ should be greater than the power of IoV $n-1$ to guarantees the successful SIC decoding at receivers. Since SIC is very critical for NOMA performance, its successful execution depends on the power levels of IoVs. The power of IoVs associated with SAP $m$ should satisfy as

$$
\left(p_{n, m}-\sum_{l=1}^{n-1} p_{l, m}\right)\left|g_{n-1}\right|^{2} \geq \omega, \forall m
$$

where $p_{n, m}$ and $p_{l, m}$ are the transmit power of SAP $m$ for IoV $n$ and $\operatorname{IoV} l$, and $\omega$ denotes the ratio of power difference which depends on hardware sensitivity and channel conditions. The SAP $m$ superimposed all the signals of its serving IoVs using superposition coding approach. Thus, the transmitted signal of SAP $m$ can be written as $x_{m}=\sum_{n=1}^{N} \sqrt{p_{n, m}} x_{n, m}$, where $x_{n, m}$ is the unit-power data symbol of IoV $n$. Based on the proposed system model and the above observations, a received 
signal at IoV $n$ from SAP $m$ can be given as

$$
\begin{aligned}
& y_{n, m}=g_{n, m} \sqrt{p_{n, m}} x_{n, m}+g_{n, m} \sum_{l=1}^{n-1} \sqrt{p_{l, m}} x_{l, m} \\
& +\beta \sum_{l=1}^{n-1} \sqrt{p_{l, m}} x_{l, m}+\sum_{m^{\prime}=1}^{M} g_{n, m}^{m^{\prime}} \sum_{n^{\prime}=1}^{N} \sqrt{p_{n^{\prime}, m^{\prime}}} x_{n^{\prime}, m^{\prime}}+\omega_{n, m},
\end{aligned}
$$

where the first term is the unit-power desired signal of IoV $n$ from the SAP $m$, the second term denotes the intra-cell interference at IoV $n$ from $n-1$ IoVs after the SIC decoding process. The third term is interference due to imperfect SIC decoding, where $\beta$ shows the imperfect SIC parameter. The fourth term is the inter-cell interference from the neighboring SAPs duce to co-channel while the last term represents the AWGN of IoV $n$ having $\sigma^{2}$ variance. Based on (3) of IoV $n$, the instantaneous signal to interference plus noise ratio (SINR) at IoV $n$ from the SAP $m$ can stated as

$$
\gamma_{n, m}=\frac{p_{n, m}\left|g_{n, m}\right|^{2}}{\sigma^{2}+\left|g_{n, m}\right|^{2} \sum_{l=1}^{n-1} p_{l, m}+\Phi_{n, m}+\left|g_{n, m}^{m^{\prime}}\right|^{2} \Theta_{n, m}} .
$$

where $\Phi_{n, m}=\left(\left|g_{n, m}\right|^{2} \sum_{j=n+1}^{N} p_{j, m}\right) \beta$ is the interference due to imperfect SIC decoding, where $\beta=\mathbb{E}\left[\left|x_{n, m}-\tilde{x}_{n, m}\right|^{2}\right]$. Note that $x_{n, m}-\tilde{x}_{n, m}$ is the difference of actual and estimated signals.

The main objective of this work is to investigate the tradeoff on the capacity and energy of NOMA SVNet. In particular, we simultaneously maximize the total achievable capacity and minimize the total energy consumption through joint optimization of power allocation. It is achieved through multi-objective optimization. A multi-objective problem is mathematically formulated as

$$
\begin{aligned}
\mathrm{P}_{1} & \max _{p_{n}, m} \sum_{m=1}^{M} \sum_{n=1}^{N} C_{n, m} \\
& \min _{p_{n, m}} \sum_{m=1}^{M} \sum_{n=1}^{N} p_{m, n} \\
\text { s.t. } & \sum_{m=1}^{M} C_{n, m} \geq C_{m i n}, \forall n \in N, \\
& \sum_{m=1}^{M} p_{n, m} \leq P_{m}, \forall n \in N, \\
& \sum_{m=1}^{M}\left(p_{n, m}-\sum_{l=1}^{n-1} p_{l, m}\right)\left|g_{n-1}\right|^{2} \geq \omega, \forall n \in N, \\
& p_{n, m} \geq 0, \forall n \in N, \forall m \in M,
\end{aligned}
$$

where $C_{n, m}=\log _{2}\left(1+\gamma_{n, m}\right)$ in $\mathrm{P}_{1}$ is the instantaneous achievable capacity of IoV $n$ from SAP $m$. The term $P_{m}$ is the total power budget of SAP $m$ and $C_{m i n}$ denotes the minimum threshold of achievable capacity to guarantee the QoS of each IoV. Moreover, (5) and (6) are the objectives of total achievable capacity maximization and total power consumption minimization. Constraint in $\left(C^{\prime} 1\right)$ guarantees the minimum achievable capacity of each IoV. Constraint in $\left(\mathrm{C}^{\prime} 2\right)$ controls the power of each SAP. Constraint in $\left(\mathrm{C}^{\prime} 3\right)$ ensures efficient signal decoding at IoVs. Constraint in $\left(\mathrm{C}^{\prime} 4\right)$ keeps the power of each IoV non-negative.

\section{Proposed Optimal Solution}

The joint power allocation problem $\mathrm{P}_{1}$ is nonlinear due to the interference terms in (5) and $\left(C^{\prime} 1\right)$ [36]. Thus, we first transform the multi-objective optimization problem by adopting the weighted-sum method and then exploit the SQP method to obtain the optimal solutions. The weighted-sum method is considered to be one of the powerful approaches for handling multi-objective problems [37], [38]. With the help of this method, any problem with multiple objectives can be linearly converted into a single-objective optimization problem. Then, a weighted coefficient that investigates a trade-off among various objectives is applied. Following this method, the formulated optimization problem $\mathrm{P}_{1}$ can be transformed as $\mathrm{P}_{2}$

$$
\begin{array}{ll}
\mathrm{P}_{2} \max _{p_{n, m}} \ell \sum_{m=1}^{M} \sum_{n=1}^{N} C_{n, m}-(1-\ell) \sum_{m=1}^{M} \sum_{n=1}^{N} p_{n, m} \\
\text { s.t. } & \left(\mathbf{C}^{\prime} 1\right),\left(\mathbf{C}^{\prime} 2\right) . \\
& \sum_{m=1}^{M}\left(\sum_{l=1}^{n-1} p_{l, m}+\frac{\omega}{\left|g_{n-1}\right|^{2}} \leq p_{n, m}\right), \forall n \in N,
\end{array}
$$

where $\ell$ is the weighted coefficient and its values lie between zero and one such as $0 \leq \ell \leq 1$. It is important to note that the higher values of $\ell$ help the total achievable capacity and its lower values support the energy efficiency of SVNet. Thus, depends on the nature of different applications and their requirements, it is the network's responsibility to select the desired values. For example, if achievable capacity is the requirement of the application, higher values of $\ell$ will be chosen. On the other side, if the energy consumption is crucial to application, then lower values of $\ell$ will be selected. Besides, constraint $\left(\mathrm{C}^{\prime} 5\right)$ is obtained from $\left(\mathrm{C}^{\prime} 3\right)$.

The optimization problem (7) is still nonlinear, thus, we exploit the SQP approach which is one of the powerful tools for solving the nonlinear power allocation problems [39]. This is the iterative approach where the nonlinear optimization problem can be modeled by quadratic optimization subproblems [40]. By employing the SQP method, the first step is to define a Jacobian matrix such as

$$
A=\left[\begin{array}{c}
{\left[\frac{\partial C_{\min }(p)}{\partial p}\right]_{N \times N}^{T}\left[\frac{\partial P_{m}(p)}{\partial p}\right]_{N \times N}^{T}} \\
{\left[\left[\frac{\partial \omega_{1}(p)}{\partial p}\right]_{N \times 1}^{T}\left[\frac{\partial \omega_{o}(p)}{\partial p}\right]_{N \times(N-1)}^{T}\right]_{N \times N}^{T}}
\end{array}\right]^{T}, \forall m
$$

where

$$
\begin{aligned}
C_{\min }(p)= & {\left[C_{\min }-C_{1, m}, C_{\min }-C_{2, m},\right.} \\
& \left.\ldots, C_{\min }-C_{n, m}, \ldots, C_{\min }-C_{N, m}\right]^{T},
\end{aligned}
$$


TABLE I: Values of (21) and (22)

\begin{tabular}{|c|c|}
\hline$D_{n, m}$ & $\left|g_{n, m}\right|^{2} \sum_{l=1}^{n-1} p_{l, m}+\sigma^{2}+p_{n, m}\left|g_{n, m}\right|^{2}+\Phi_{n, m}+\left|g_{n, m}^{m^{\prime}}\right|^{2} \Theta_{n, m}$ \\
\hline$E_{l, m}$ & $2 p_{l, m}\left|g_{l, m}\right|^{4}\left(2\left(\left|g_{l, m}\right|^{2} \sum_{r=1}^{l-1} p_{r, m}+g_{l, m}^{m^{\prime}} \Theta_{l, m}\right)\left(2\left|g_{l, m}\right|^{2}\right)+2 p_{l, m}\left|g_{l, m}\right|^{4}+\Phi_{l, m}\right)$ \\
\hline$F_{l^{\prime}, m^{\prime}}$ & $p_{l^{\prime}, m^{\prime}}\left|g_{l^{\prime}, m^{\prime}}\right|^{4}\left(2\left(\left|g_{l^{\prime}, m^{\prime}}\right|^{2} \sum_{r^{\prime}=1}^{l^{\prime}-1} p_{r^{\prime}, m^{\prime}}+\left|g_{r^{\prime}, m^{\prime}}^{m^{\prime \prime}}\right|^{2} \Theta_{r^{\prime}, m^{\prime}}\right)\left(\left|g_{r^{\prime}, m^{\prime}}\right|^{2}\right)+p_{r^{\prime}, m^{\prime}}\left|g_{r^{\prime}, m^{\prime}}\right|^{2}+\Phi_{l^{\prime}, m^{\prime}}\right)$ \\
\hline$G_{k, m}$ & $\left(\left|g_{k, m}\right|^{2} \sum_{u=1}^{k-1} p_{u, m}+\Phi_{k, m}+\left|g_{k, m}^{m^{\prime}}\right|^{2} \Theta_{k, m}\right)^{2}\left(\left|g_{k, m}\right|^{2} \sum_{u=1}^{k-1} p_{u, m}+\Phi_{k, m}+\left|g_{k, m}^{m^{\prime}}\right|^{2} \Theta_{k, m}\right) p_{k, m}\left|g_{k, m}\right|^{2}$ \\
\hline$H_{k^{\prime}, m^{\prime}}$ & $\left(\left|g_{k^{\prime}, m^{\prime}}\right|^{2} \sum_{u^{\prime}=1}^{k^{\prime}-1} p_{u^{\prime}, m^{\prime}}+\Phi_{k^{\prime}, m^{\prime}}+\left|g_{u^{\prime}, m^{\prime}}^{m^{\prime \prime}}\right|^{2} \Theta_{k^{\prime}, m^{\prime}}\right)^{2}+\left(\left|g_{k^{\prime}, m^{\prime}}\right|^{2} \sum_{u^{\prime}=1}^{k^{\prime}-1} p_{u^{\prime}, m^{\prime}}+\Phi_{k^{\prime}, m^{\prime}}+\left|g_{k^{\prime}, m^{\prime}}^{m^{\prime \prime}}\right|^{2} \Theta_{k^{\prime}, m^{\prime}}\right) p_{k^{\prime}, m}\left|g_{k^{\prime}, m^{\prime}}\right|^{2}$ \\
\hline
\end{tabular}

$$
\begin{gathered}
\omega(p)=\left[0, p_{1, m}+\frac{\omega}{\left|h_{1, m}\right|^{2}}-p_{2, m},\right. \\
\left.\ldots, \sum_{l=1}^{n-1} p_{l, m}+\frac{\omega}{\left|g_{n-1}\right|^{2}} \leq p_{n, m}\right]^{T}, \\
P_{m}(p)=\left[p_{1, m}-P_{m}, p_{2, m}-P_{m}, \ldots, p_{N, m}-P_{m}\right]^{T},
\end{gathered}
$$

After calculating the partial derivations of (9), it can be written as

$$
\frac{\partial C_{\min }(p)}{\partial p_{n, m}}=\left\{\begin{array}{cl}
\Upsilon_{1}, & \text { if } f=z \\
\Upsilon_{2}, & \text { if } f>z \\
0, & \text { if } f<z
\end{array}\right.
$$

where $f$ and $z$ represents the columns and rows of matrix (12). Moreover, the values of $\Upsilon_{1}$ and $\Upsilon_{2}$ are stated as

$$
\begin{aligned}
& \Upsilon_{1}=\frac{-\left|g_{n, m}\right|^{2}}{\Lambda_{n, m}+p_{n, m}\left|g_{n, m}\right|^{2}+\Phi_{n, m}+\left|g_{n, m}^{m^{\prime}}\right|^{2} \Theta_{n, m}} \\
& \Upsilon_{2}=\frac{p_{i, m}\left|g_{i, m}\right|^{4}}{\Lambda_{i, m}\left(\Lambda_{i, m}+p_{i, m}\left|g_{i, m}\right|^{2}\right)+\Phi_{i, m}+\left|g_{i, m}^{m^{\prime}}\right|^{2} \Theta_{i, m}}
\end{aligned}
$$

where $\Lambda_{n, m}=\sigma^{2}+\left|g_{n, m}\right|^{2} \sum_{l=1}^{n-1} p_{l, m}$. Similar to the (9), the values of (10) and (11) can be written as

$$
\begin{aligned}
& \frac{\partial P_{\mathrm{m}}(p)}{\partial p_{n, m}}=\left\{\begin{array}{cl}
0, & \text { if } f>z, \\
1, & \text { if } f=z, \\
0, & \text { if } f<z,
\end{array}\right. \\
& \frac{\partial \omega_{1}(\boldsymbol{p})}{\partial p_{n, m}}=[0,0, \ldots, 0]^{T}, \forall n \in N,
\end{aligned}
$$

and $\forall o>1$, it can be stated as

$$
\frac{\partial \omega_{o}(p)}{\partial p_{n, m}}=\left\{\begin{aligned}
1, & \text { if } f>z, \\
-1, & \text { if } f=z, \\
0, & \text { if } f<z
\end{aligned}\right.
$$

Now we define the Lagrange function of (7) such as

$$
\begin{aligned}
& L\left(p_{n, m}, \lambda_{n, m}, v_{n, m}, \pi_{n, m}\right)=\ell \sum_{m=1}^{M} \sum_{n=1}^{N} C_{n, m} \\
& -(1-\ell) \sum_{m=1}^{M} \sum_{n=1}^{N} p_{n, m}+\sum_{m=1}^{M} \sum_{n=1}^{N} \lambda_{n, m}\left(C_{m i n}-C_{n, m}\right) \\
& +\sum_{m=1}^{M} v_{n, m}\left(\sum_{l=1}^{n-1} p_{l, m}+\frac{\omega}{\left|g_{n-1}\right|^{2}}-p_{n, m}\right) \\
& +\sum_{m=1}^{M} \sum_{n=1}^{N} \pi_{n, m}\left(p_{n, m}-P_{m}\right)
\end{aligned}
$$

where $\lambda_{n, m}, v_{n, m}$ and $\pi_{n, m}$ are the Lagrange multipliers. Next we derive a Hessian matrix such that its $i$ row and $j$ column can be formulated as

$$
B=\left[\begin{array}{cccc}
\frac{\partial^{2} L(.)}{\partial^{2} p_{1, m}} & \frac{\partial^{2} L(.)}{\partial p_{1, m} \partial p_{2, m}} & \cdots & \frac{\partial^{2} L(.)}{\partial p_{1, m} \partial p_{N, m}} \\
\frac{\partial^{2} L(.)}{\partial p_{2, m} \partial p_{1, m}} & \frac{\partial^{2} L(.)}{\partial^{2} p_{2, m}} & \cdots & \frac{\partial^{2} L(.)}{\partial p_{2, m} \partial p_{N, m}} \\
\vdots & \vdots & \ddots & \vdots \\
\frac{\partial^{2} L(.)}{\partial p_{N, m} \partial p_{1, m}} & \frac{\partial^{2} L(.)}{\partial p_{N, m} \partial p_{2, m}} & \cdots & \frac{\partial^{2} L(.)}{\partial^{2} p_{N, m}}
\end{array}\right]_{N \times N},
$$

The entries of (19) after partial derivations can be stated as

$$
B=\left\{\begin{array}{ll}
\Upsilon_{3}, & \text { if } f \geq z, \\
\Upsilon_{4}, & \text { if } f<z,
\end{array},\right.
$$

where the values of $\Upsilon_{3}$ and $\Upsilon_{4}$ (20) are defined as

$$
\begin{aligned}
& \Upsilon_{3}=\frac{\left|g_{n, m}\right|^{4}}{D_{n, m}^{2}}-\sum_{l=1}^{n-1} \frac{E_{l, m}}{G_{l, m}^{2}}-\sum_{m=1}^{M} \sum_{n^{\prime}=1}^{N} \frac{F_{n^{\prime}, m^{\prime}}}{H_{n^{\prime}, m^{\prime}}^{2}}, \\
& \Upsilon_{4}=\frac{E_{k, m}}{G_{k, m}^{2}}-\sum_{k=1}^{l-1} \frac{E_{k, m}}{G_{k, m}^{2}}-\sum_{m^{\prime}=1}^{M} \sum_{k^{\prime}=1}^{N} \frac{F_{k^{\prime}, m^{\prime}}}{H_{k^{\prime}, m^{\prime}}^{2}} .
\end{aligned}
$$

and $D_{n, m}, E_{l, m}, F_{l^{\prime}, m^{\prime}}, G_{k, m}, H_{k^{\prime}, m^{\prime}}$, are stated in TABLE I at the top of the page. Finally, we define matrix $C$ such that

$$
C=\left[\begin{array}{l}
{[B]_{N \times N}[A]_{N \times 3 N}^{T}} \\
{[A]_{3 N \times N}[0]_{3 N \times 3 N}}
\end{array}\right]_{4 N \times 4 N},
$$

We improve the estimate of $\left(p_{n, m}, \lambda_{n, m}, v_{n, m}, \pi_{n, m}\right)$ iteratively such as

$$
\phi^{t+1}=\phi^{t}+\alpha \psi^{t},
$$

where $\alpha$ is the step-size and $\phi$ can be derived as $\phi=$ $\left[p^{t+1}, \lambda^{t+1}, v^{t+1}, \pi^{t+1}\right]^{T}$. Moreover, the $\psi$ is the correction 
vector as $\psi=\left[\left(\psi_{p}^{t}\right)^{T},\left(\psi_{\lambda}^{t}\right)^{T},\left(\psi_{v}^{t}\right)^{T},\left(\psi_{\pi}^{t}\right)^{T}\right]^{T}$ and can be calculated as

$$
\psi=\left[\zeta L(p)^{T}, \zeta L(\lambda)^{T}, \zeta L(v)^{T}, \zeta L(\pi)^{T}\right]^{T} \times-C^{-1},
$$

where $\zeta L(p), \zeta L(\lambda), \zeta L(v)$, and $\zeta L(\pi)$ are the Gradient obtained from the Lagrangian function and can be expressed as [41]

$$
\begin{gathered}
\zeta L\left(p_{n, m}\right)=-\left(1+\lambda_{n, m}\right) \Upsilon_{5}+\sum_{l=1}^{n-1} \lambda_{l, m} \Upsilon_{6}-v_{n, m}+\pi_{n, m} \\
\zeta L\left(\lambda_{n, m}\right)=\sum_{m=1}^{M}\left(C_{m i n}-C_{n, m}\right), \forall n \\
\zeta L\left(v_{n, m}\right)=\sum_{m=1}^{M}\left(\sum_{l=1}^{n-1} p_{l, m}+\frac{\omega}{\left|g_{n-1}\right|^{2}}-p_{n, m}\right), \forall n \\
\zeta L\left(\pi_{n, m}\right)=\sum_{m=1}^{M}\left(p_{n, m}-P_{m}\right), \forall n
\end{gathered}
$$

where the values of $\Upsilon_{5}$ and $\Upsilon_{6}$ can be written as

$$
\begin{aligned}
& \Upsilon_{5}=\frac{\left|g_{n, m}\right|^{2}}{\Lambda_{n, m}+p_{n, m}\left|g_{n, m}\right|^{2}+\Phi_{n, m}+\left|g_{n, m}^{m^{\prime}}\right|^{2} \Theta_{n, m}}, \\
& \Upsilon_{6}=\frac{p_{j, m}\left|g_{j, m}\right|^{4}}{\Lambda_{j, m}\left(\Lambda_{j, m}+p_{j, m}\left|g_{j, m}\right|^{2}\right)+\Phi_{j, m}+\left|g_{j, m}^{m^{\prime}}\right|^{2} \Theta_{j, m}}
\end{aligned}
$$

The complexity to solve the proposed SVNet framework using the SQP method in terms of iteration depends on $M$ and $N$. If the number of iterations required for convergence is $T$, the total computational complexity of the proposed framework can be computed as $\mathcal{O}\left(T M N^{2}\right)$. The complete steps of the joint power optimization method are also discussed in Algorithm 1.

\section{Results And Discussion}

In this section, we present and discuss the simulation results of our proposed NOMA SVNet approach. We obtain the average results from $10^{4}$ trials using Monte Carlo simulations. We compare the proposed optimization approach with benchmark NOMA SVNet, average power NOMA SVNet and conventional OMA SVNet. The benchmark NOMA SVNet refers to a traditional optimization approach using KKT conditions, average power NOMA approach uses the arithmetic progression technique such that the power of IoVs in each cell should satisfy as $p_{n, m}=\frac{2 n}{N(1+N)} P_{m}$, and the conventional OMA approach accommodates only one IoV with each SAP at any given time. Unless otherwise mentioned, the simulation parameters are set as: The number of SAPs is $M=6$, the number of IoVs associated with each SAP at any given time is $N=2$, the maximum power budget of SAP is set as $P_{m}=35 \mathrm{dBm}$, the values of weighted coefficient $\ell$ to calculate the trade-off between energy efficiency and sum-capacity are set as $\ell=$ $0.1,0.2,0.3,0.4,0.5,0.6,0.7,0.8,0.9,1$, the values of impSIC
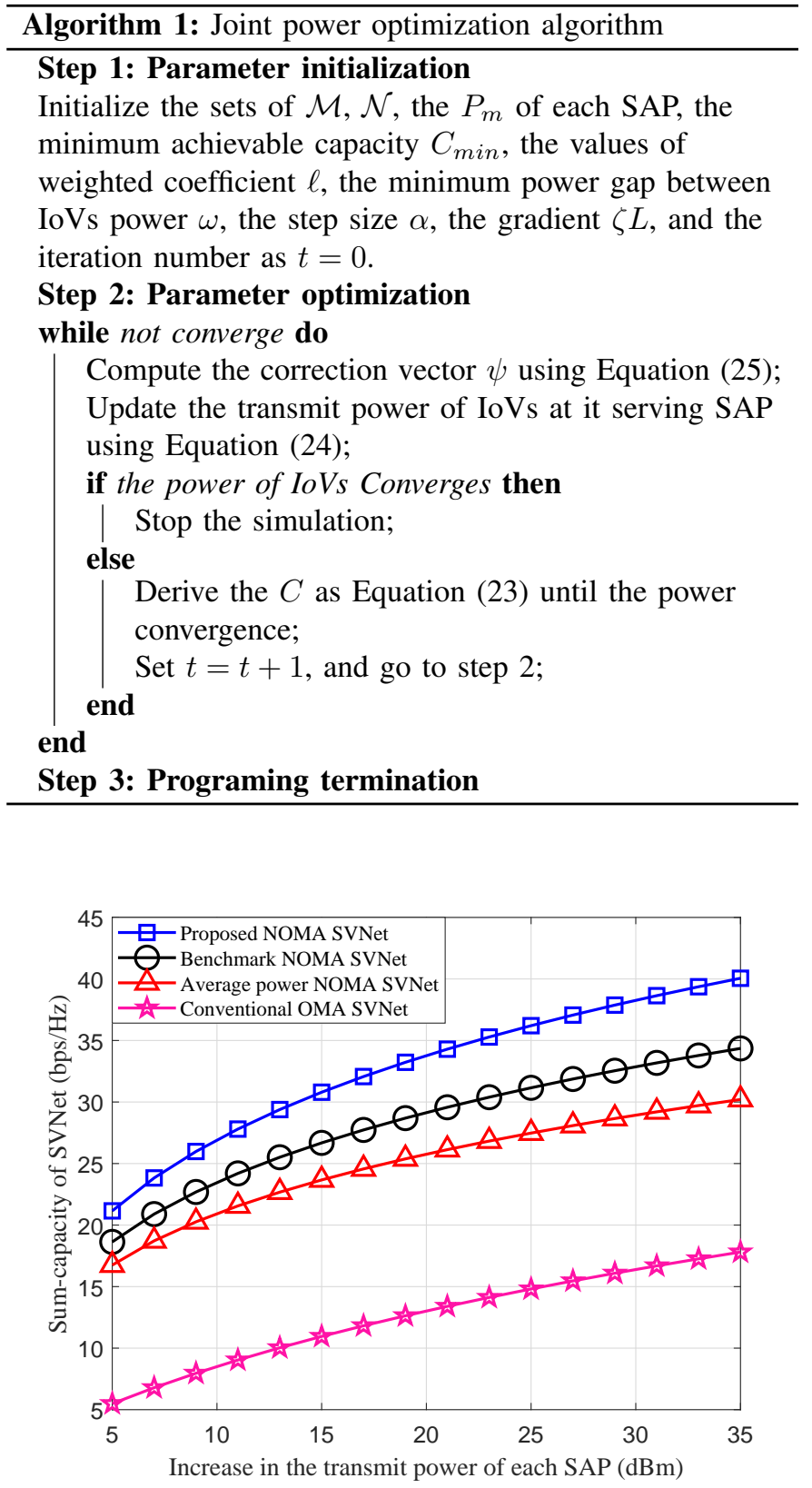

Fig. 2: Sum-capacity of SVNet versus increase in the transmit power of each SAP for $M=6, \ell=1, \Phi=0.1$ and $C_{m i n}=1.5 \mathrm{bps} / \mathrm{Hz}$.

parameter are $\Phi_{n, m}=0.1,0.2,0.3,0.4,0.5,0.6,0.7,0.8$ the variance is set as $\phi=0.1$, the ratio of power different for efficient SIC decoding is set as $2 \mathrm{dBm}$, the circuit power $p_{c}$ is set as $p_{c}=10 \mathrm{dBm}$, and the wireless channels undergo Rayleigh fading. In addition, the pathloss is $\varepsilon=128.1+37.6 \log (d)$ [32], and the minimum capacity threshold of each IoV is set as: $1.5 \mathrm{bps} / \mathrm{Hz}$ when $\xi=1,0.9,0.8,0.7,0.6,0.5$, and $1 \mathrm{bps} / \mathrm{Hz}$ when $\xi=0.4,0.3,0.2$, respectively.

First, we show the impact of SAP's transmit power on the sum-capacity and total energy efficiency of SVNet in Figs. 2 and 3 which depict the sum-capacity and total energy efficiency against the transmit power of SAPs. In both figures, the transmit power of each SAP varies from 5 to 35 $\mathrm{dBm}$. Moreover, the total energy efficiency of the SVNet is 


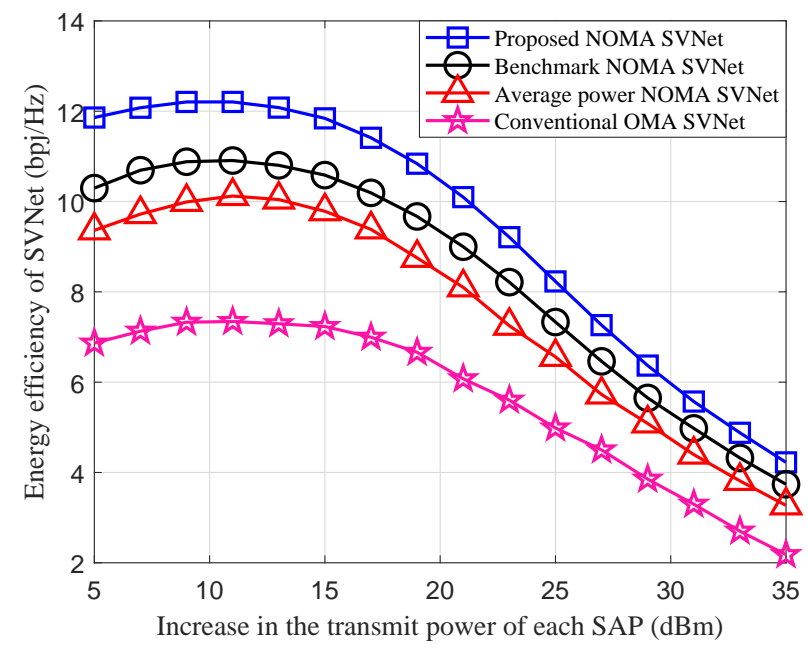

Fig. 3: Energy efficiency of SVNet versus increase in the transmit power of each SAP for $M=6, p_{c}=10 \mathrm{dBm}, \ell=0.3, \Phi=0.1$ and $C_{\min }=1 \mathrm{bps} / \mathrm{Hz}$.

calculated as

$$
E E=\sum_{m=1}^{M} \sum_{n}^{N} \frac{\log _{2}\left(1+\gamma_{n, m}\right)}{P_{n, m}+p_{c}}, \mathrm{bpj} / \mathrm{Hz} .
$$

Fig. 2 plots the sum-capacity versus the transmit power of each SAP. We can see that the sum-capacity of the proposed NOMA SVNet, and other approaches increases with the increasing transmit power of SAP. It can also be evident that the proposed SVNet approach performs significantly better than the other baseline SVNet approaches. For instance, when the transmit power of each SAP is $31 \mathrm{dBm}$, the sumcapacity of the proposed SVNet is $38.64 \mathrm{bps} / \mathrm{Hz}$ compared to other approaches which only achieve $33.18,29.20$, and 16.69 $\mathrm{bps} / \mathrm{Hz}$, respectively. Moreover, it is worth mentioning that the gap of sum-capacity between the proposed approach and other approaches increases when the transmit power of SAP increases. It is because the proposed approach is more efficient when SAPs are transmitting at high power.

Fig. 3 discusses the importance of transmit power on the total energy efficiency of SVNet. It is demonstrated that the energy efficiency of all SVNet approaches decreases with the increasing transmit power of SAP. We can also observe that the energy efficiency of the proposed approach and other approaches follows a bell-shaped curve where it first increases as the transmit power per SAP increases until saturating point and then it falls decreasing with further increase in transmit power. However, the proposed NOMA SVNet approach performs better compared to other approaches. For example, when the system operates over $10 \mathrm{dBm}$ transmit power of each SAP, the energy efficiency of the proposed NOMA SVNet approach is $12.21 \mathrm{bpj} / \mathrm{Hz}$, whereas benchmark NOMA SVNet, average power NOMA SVNet and conventional OMA SVNet achieve only $10.90,10.10$, and $7.3 \mathrm{bpj} / \mathrm{Hz}$. It can also be noted that the performance gap between NOMA approaches and the OMA approach is large, which shows its poor performance.

Then, we describe the importance of the number of SAPs on the sum-capacity and total energy efficiency of SVNet. In

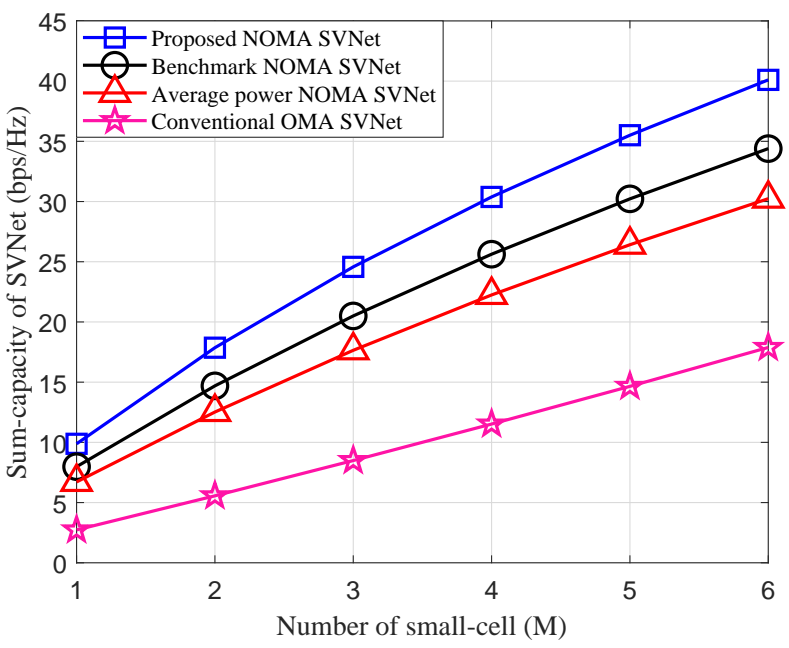

Fig. 4: Sum-capacity of SVNet versus the number of small-cell for $P_{m}=35 \mathrm{dBm}, \ell=1, \Phi=0.1$ and $C_{\min }=1.5 \mathrm{bps} / \mathrm{Hz}$.

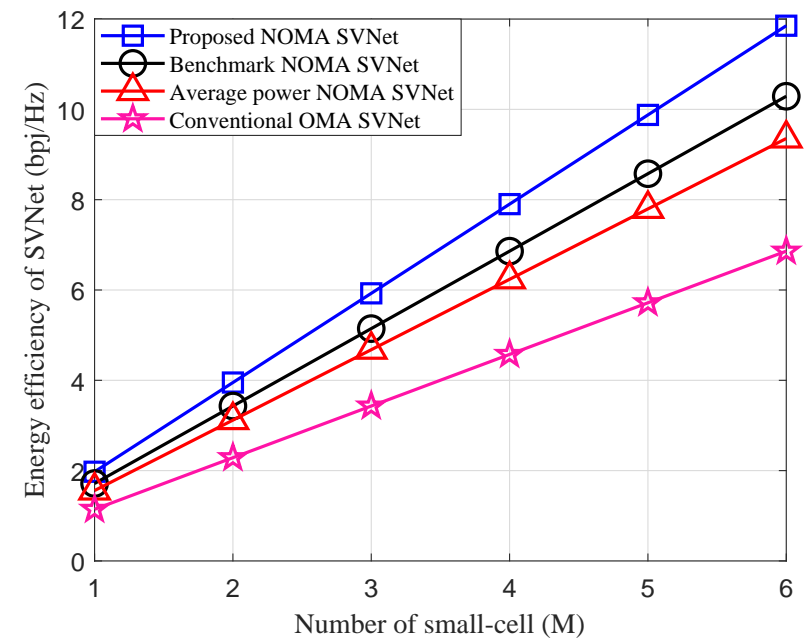

Fig. 5: Energy efficiency of SVNet versus the number of small-cell for $P_{m}=35 \mathrm{dBm}, p_{c}=10 \mathrm{dBm}, \ell=0.3, \Phi=0.1$ and $C_{m i n}=1$ $\mathrm{bps} / \mathrm{Hz}$.

this regard, Figs. 4 and 5 plot the sum-capacity and energy efficiency against the increasing number of SAPs in the system which varies from 1 to 6 .

Fig. 4 illustrates the sum-capacity versus the number of $S$ APs. We can see that the sum-capacity of all SVNet approaches increases as the number of SAPs increases. It is because more IoVs can be accommodated by the same frequency and time resources. As expected, the proposed NOMA SVNet approach outperforms the other SVNet approaches. For instance, if the number of SAP is 6, the proposed SVNet achieves $40.10 \mathrm{bps} / \mathrm{Hz}$. However, for the same point, the other SVNet approaches obtain 34.39, 30.24, and $17.88 \mathrm{bps} / \mathrm{Hz}$. Moreover, the performance between the proposed approach and other approaches increases as the number of SAPs increases which indicates that the proposed approach is more suitable for largescale IoVs.

Fig. 5 describes the system's total energy efficiency versus 


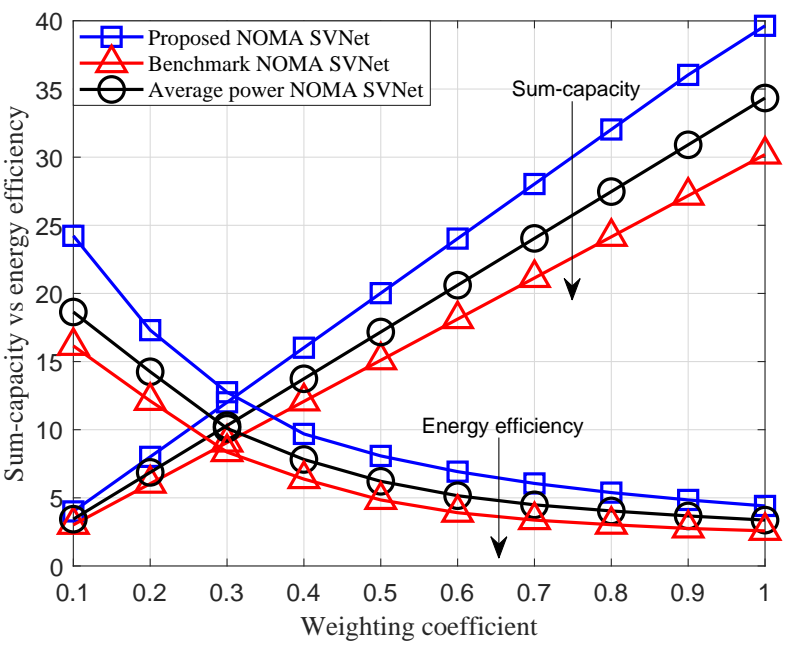

Fig. 6: The impact of weighting coefficient sum-capacity and energy efficiency for $M=6, P_{m}=35 \mathrm{dBm}, \Phi=0.1, p_{c}=10 \mathrm{dBm}$, and $C_{\min }=1.5 \mathrm{bps} / \mathrm{Hz}$.

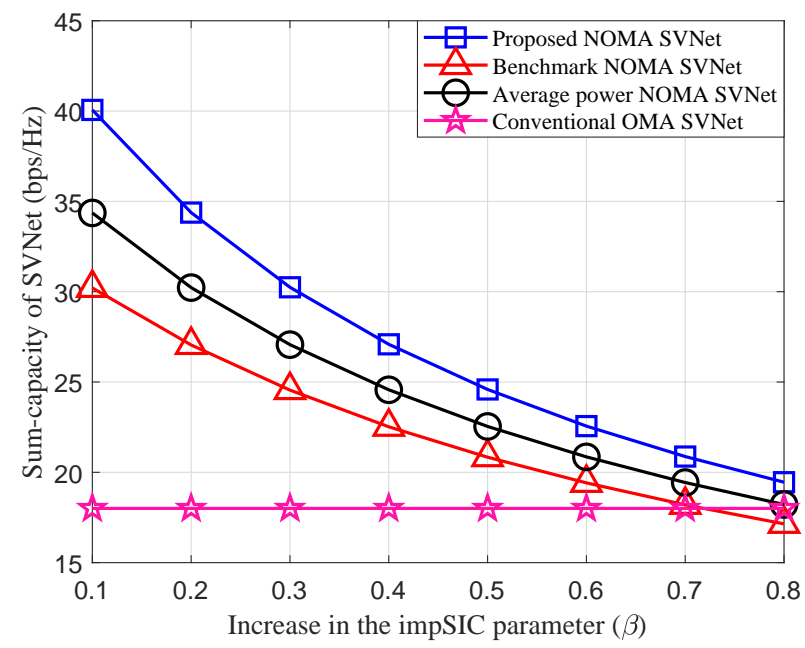

Fig. 7: Increase in the impSIC parameter versus the sum-capacity of SVNet for $M=6, P_{m}=35 \mathrm{dBm}, \ell=1$, and $C_{\text {min }}=1 \mathrm{bps} / \mathrm{Hz}$.

the number of SAPs. It can be observed that the energy efficiency of all approaches increases as the number of SAPs increases. However, the proposed NOMA SVNet approach achieves more energy efficiency compared to other SVNet approaches. For example, the energy efficiency of the proposed approaches is 11.85 when the number of SAPs reaches 6 . At the same point, the energy efficiency of the other SVNet approaches is respectively, 10.29, 9.35, and $6.86 \mathrm{bpj} / \mathrm{Hz}$. Furthermore, one can also be noted that the energy efficiency gap between the proposed approach and other SVNet approaches increases when the number of SAPs increases which shows the effectiveness of our approach for large-scale networks.

Next, it is important to study the trade-off between sumcapacity and energy efficiency. Fig. 6 analyses the sumcapacity and energy efficiency versus the varying values of weighting coefficient for the proposed NOMA SVNet approach and other NOMA approaches. As expected, the sum-rate increases and energy efficiency decreases with the increasing values of the weighting coefficient. The proposed NOMA SVNet approach outperforms the other approaches in both sum-capacity and energy efficiency. It is important to mention here that the high values of the weighting coefficient help sum-capacity and its lower values support energy efficiency. Thus, depends on the nature of different applications and their requirements, it is the network's responsibility to select the desired values. For example, if high sum-capacity is the requirement of the application, the high value of the weighting coefficient will be chosen. On the contrary, if energy consumption is crucial to application, then lower values of the weighting coefficient will be selected.

Now we study the impact of impSIC parameters on the performance of different SVNet approaches. Fig. 7 shows the sum-capacity versus increase in the impSIC parameter which varies from 0.1 to 0.8 . It is evident that the sum capacity of all approaches decreases when the values of impSIC parameter increase. It shows the importance of perfect SIC decoding in practical systems. The proposed SVNet approach still obtains high sum-capacity than the other benchmark approaches. Moreover, we can see no changes in the performance of the conventional SVNet approach. It is because the optimization of the OMA system is independent of the values of impSIC parameter.

Finally, Fig. 8 discusses the effects of circuit power on the system performance by plotting the energy efficiency against the increasing values of circuit power for all the considered approaches. We can note that the energy efficiency of SVNet decreases as the circuit power increases. Apparently, the conventional OMA SVNet approach consumes high power for efficient transmission rapidly reducing energy efficiency compared to the NOMA SVNet approaches. The proposed NOMA SVNet approach achieves high energy efficiency for a large-scale of energy-constrained IoVs.

\section{CONCLUSiOnS}

NOMA and IoVs are the key enablers for low-powered large-scale 6G intelligent transportation systems. This paper has provided a multi-objective optimization framework for NOMA SVNet under imperfect SIC. In particular, the sumcapacity and energy efficiency of SVNet have been simultaneously maximized through joint power optimization. A new iterative approach based on SQP has been exploited to solve the nonlinear power allocation problem. Presented results show that the proposed NOMA SVNet performs better than the other benchmark approaches in system sum-capacity and total energy efficiency. The proposed optimization framework can be extended in several ways. For example, we can extend it by incorporating the channel estimation errors. Afterward, we also aim to extend them by incorporating backscatter communication in the considered system model which can further increase total energy efficiency. These interesting open issues will be addressed in our future studies.

\section{REFERENCES}

[1] F. Jameel, W. U. Khan, N. Kumar, and R. Jntti, "Efficient powersplitting and resource allocation for cellular V2X communications," 


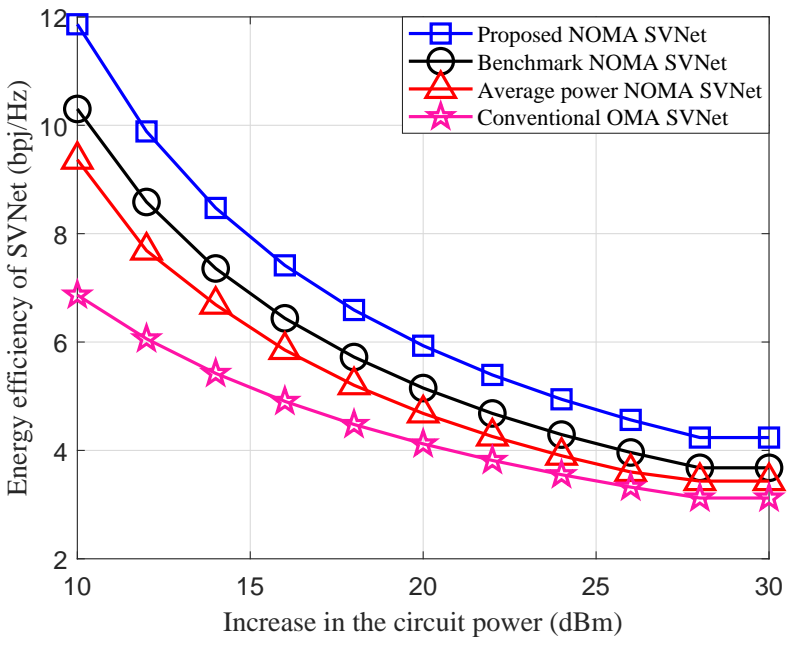

Fig. 8: Impact of circuit power on the energy efficiency of SVNet for $M=6, P_{m}=35 \mathrm{dBm}, \ell=0.3, \Phi=0.1$ and $C_{\min }=1 \mathrm{bps} / \mathrm{Hz}$.

IEEE Transactions on Intelligent Transportation Systems, pp. 1-10, 2020.

[2] S. Safavat and D. B. Rawat, "On the elliptic curve cryptography for privacy-aware secure ACO-AODV routing in intent-based internet of vehicles for smart cities," IEEE Transactions on Intelligent Transportation Systems, pp. 1-10, 2020.

[3] A. U. Makarfi, K. M. Rabie, O. Kaiwartya, K. Adhikari, G. Nauryzbayev, $\mathrm{X}$. Li, and R. Kharel, "Toward physical-layer security for internet of vehicles: Interference-aware modeling," IEEE Internet of Things Journal, vol. 8, no. 1, pp. 443-457, Jan. 2021.

[4] P. K. Singh, R. Singh, S. K. Nandi, K. Z. Ghafoor, D. B. Rawat, and S. Nandi, "Blockchain-based adaptive trust management in internet of vehicles using smart contract," IEEE Transactions on Intelligent Transportation Systems, pp. 1-15, 2020.

[5] F. Jameel, S. Wyne, M. A. Javed, and S. Zeadally, "Interference-aided vehicular vetworks: Future research opportunities and challenges," IEEE Communications Magazine, vol. 56, no. 10, pp. 36-42, Oct. 2018.

[6] M. Abbasi, A. Najafi, M. Rafiee, M. R. Khosravi, V. G. Menon, and G. Muhammad, "Efficient flow processing in 5G-envisioned SDN-based internet of vehicles using GPUs," IEEE Transactions on Intelligent Transportation Systems, pp. 1-10, 2020.

[7] F. Tang, Y. Kawamoto, N. Kato, and J. Liu, "Future intelligent and secure vehicular network toward 6G: Machine-learning approaches," Proceedings of the IEEE, vol. 108, no. 2, pp. 292-307, Feb. 2020.

[8] W. U. Khan, J. Liu, F. Jameel, V. Sharma, R. Jantti, and Z. Han, "Spectral efficiency optimization for next generation NOMA-enabled IoT networks," IEEE Transactions on Vehicular Technology, pp. 1-1, 2020.

[9] X. Li, M. Zhao, M. Zeng, S. Mumtaz, V. G. Menon, Z. Ding, and O. A. Dobre, "Hardware impaired ambient backscatter NOMA systems: Reliability and security," arXiv preprint arXiv:2008.05798, 2020.

[10] W. U. Khan, X. Li, M. Zeng, and O. A. Dobre, "Backscatter-enabled NOMA for future 6G systems: A new optimization framework under imperfect SIC," IEEE Communications Letters, pp. 1-1, 2021.

[11] X. Li, Q. Wang, M. Liu, J. Li, H. Peng, J. Piran, and L. Li, "Cooperative wireless-powered NOMA relaying for B5G IoT networks with hardware impairments and channel estimation errors," IEEE Internet of Things Journal, pp. 1-1, 2020.

[12] W. U. Khan, F. Jameel, T. Ristaniemi, S. Khan, G. A. S. Sidhu, and J. Liu, "Joint spectral and snergy efficiency optimization for downlink NOMA networks," IEEE Transactions on Cognitive Communications and Networking, vol. 6, no. 2, Jun. 2020.

[13] X. Li, M. Zhao, Y. Liu, L. Li, Z. Ding, and A. Nallanathan, "Secrecy analysis of ambient backscatter NOMA systems under I/Q imbalance," IEEE Transactions on Vehicular Technology, vol. 69, no. 10, pp. 12286 12290 , Oct. 2020.

[14] F. Jameel, S. Zeb, W. U. Khan, S. A. Hassan, Z. Chang, and J. Liu, "NOMA-enabled backscatter communications: Toward battery-free IoT networks," IEEE Internet of Things Magazine, pp. 1-7, 2020.
[15] H. Zheng, H. Li, S. Hou, and Z. Song, "Joint resource allocation with weighted max-min fairness for NOMA-enabled V2X communications," IEEE Access, vol. 6, pp. 65 449-65 462, 2018.

[16] B. Wang, R. Zhang, C. Chen, X. Cheng, L. Yang, and Y. Jin, "Interference hypergraph-based 3D matching resource allocation protocol for NOMA-V2X networks," IEEE Access, vol. 7, pp. 90 789-90 800, 2019.

[17] D. Zhang, Y. Liu, L. Dai, A. K. Bashir, A. Nallanathan, and B. Shim, "Performance analysis of FD-NOMA-based decentralized V2X systems," IEEE Transactions on Communications, vol. 67, no. 7, pp. 50245036, Jul. 2019.

[18] H. Xiao, Y. Chen, S. Ouyang, and A. T. Chronopoulos, "Power control for clustering car-following V2X communication system with nonorthogonal multiple access," IEEE Access, vol. 7, pp. 68 160-68 171, 2019.

[19] B. Wang, R. Zhang, C. Chen, X. Cheng, and L. Yang, "Interference hypergraph-based resource allocation (IHG-RA) for NOMA-integrated V2X networks," in 2018 IEEE Global Communications Conference (GLOBECOM), 2018, pp. 1-6.

[20] C. Chen, B. Wang, and R. Zhang, "Interference hypergraph-based resource allocation (IHG-RA) for NOMA-integrated V2X networks," IEEE Internet of Things Journal, vol. 6, no. 1, pp. 161-170, Feb. 2019.

[21] L. P. Qian, Y. Wu, H. Zhou, and X. Shen, "Dynamic cell association for non-orthogonal multiple-access V2S networks," IEEE Journal on Selected Areas in Communications, vol. 35, no. 10, pp. 2342-2356, Oct. 2017.

[22] Y. Chen, L. Wang, Y. Ai, B. Jiao, and L. Hanzo, "Performance analysis of NOMA-SM in vehicle-to-vehicle massive MIMO channels," IEEE Journal on Selected Areas in Communications, vol. 35, no. 12, pp. $2653-$ 2666, Dec. 2017.

[23] B. Di, L. Song, Y. Li, and G. Y. Li, "NOMA-based low-latency and high-reliable broadcast communications for 5G V2X services," in GLOBECOM 2017 - 2017 IEEE Global Communications Conference, 2017, pp. 1-6.

[24] _ "Non-orthogonal multiple access for high-reliable and low-latency V2X communications in 5G systems," IEEE Journal on Selected Areas in Communications, vol. 35, no. 10, pp. 2383-2397, Oct. 2017.

[25] S. Guo and X. Zhou, "Robust power allocation for NOMA in heterogeneous vehicular communications with imperfect channel estimation," in 2017 IEEE 28th Annual International Symposium on Personal, Indoor, and Mobile Radio Communications (PIMRC), 2017, pp. 1-5.

[26] _ - "Robust resource allocation with imperfect channel estimation in NOMA-based heterogeneous vehicular networks," IEEE Transactions on Communications, vol. 67, no. 3, pp. 2321-2332, March 2019.

[27] N. Jaiswal and N. Purohit, "Performance of downlink NOMA-enabled vehicular communications over double Rayleigh fading channels," IET Communications, vol. 14, no. 20, pp. 3652-3660, 2020

[28] D. T. Do, M. S. V. Nguyen, A. T. Le, K. M. Rabie, and J. Zhang, "Joint full-duplex and roadside unit selection for NOMA-enabled V2X communications: ergodic rate performance," IEEE Access, vol. 8, pp. $140348-140360,2020$.

[29] O. Abbasi, H. Yanikomeroglu, A. Ebrahimi, and N. M. Yamchi, "Trajectory design and power allocation for drone-assisted NR-V2X network with dynamic NOMA/OMA," IEEE Transactions on Wireless Communications, vol. 19, no. 11, pp. 7153-7168, Nov. 2020.

[30] D.-T. Do, A.-T. Le, T.-A. Hoang, and B. M. Lee, "Cognitive radioassisted NOMA broadcasting for $5 \mathrm{G}$ cellular V2X communications: Model of roadside unit selection and SWIPT," Sensors, vol. 20, no. 6, p. 1786, 2020.

[31] W. U. Khan, F. Jameel, G. A. S. Sidhu, M. Ahmed, X. Li, and R. Jntti, "Multiobjective optimization of uplink NOMA-enabled vehicleto-infrastructure communication," IEEE Access, vol. 8, pp. 8446784478,2020

[32] A. Ihsan, W. Chen, S. Zhang, and S. Xu, "Energy-efficient NOMA multicasting system for $5 \mathrm{G}$ cellular V2X communications with imperfect CSI," arXiv preprint arXiv:2009.03507, 2020.

[33] W. U. Khan, F. Jameel, N. Kumar, R. Jntti, and M. Guizani, "Backscatter-enabled efficient V2X communication with non-orthogonal multiple access," IEEE Trnsactions on Vehicular Technology, pp. 1-1, 2021.

[34] Y. Liu, H. Zhang, K. Long, A. Nallanathan, and V. C. M. Leung, "Energy-efficient subchannel matching and power allocation in NOMA autonomous driving vehicular networks," IEEE Wireless Communications, vol. 26, no. 4, pp. 88-93, Aug. 2019.

[35] W. U. Khan, Z. Yu, S. Yu, G. A. S. Sidhu, and J. Liu, "Efficient power allocation in downlink multi-cell multi-user NOMA networks," IET Communications, vol. 13, no. 4, pp. 396-402, 2018. 
[36] W. U. Khan, Z. Ali, M. Waqas, and G. A. S. Sidhu, "Efficient power allocation with individual QoS guarantees in future small-cell networks," AEU-International Journal of Electronics and Communications, vol. 105 , pp. 36-41, 2019.

[37] R. T. Marler and J. S. Arora, "Survey of multi-objective optimization methods for engineering," Structural and multidisciplinary optimization, vol. 26, no. 6, pp. 369-395, 2004.

[38] _ - "The weighted sum method for multi-objective optimization: new insights," Structural and multidisciplinary optimization, vol. 41, no. 6, pp. 853-862, 2010.

[39] P. T. Boggs and J. W. Tolle, "Sequential quadratic programming for large-scale nonlinear optimization," Journal of computational and applied mathematics, vol. 124, no. 1-2, pp. 123-137, 2000.

[40] A. Attar, M. R. Nakhai, and A. H. Aghvami, "Cognitive radio game for secondary spectrum access problem," IEEE Transactions on Wireless Communications, vol. 8, no. 4, pp. 2121-2131, Apr. 2009.

[41] W. U. Khan, F. Jameel, M. A. Jamshed, H. Pervaiz, S. Khan, and J. Liu, "Efficient power allocation for NOMA-enabled IoT networks in 6G era," Physical Communication, vol. 39, p. 101043, 2020. 